\title{
Behavioural activation: history, evidence and promise
}

Jonathan W. Kanter, Ajeng J. Puspitasari, Maria M. Santos and Gabriela A. Nagy

\section{Summary}

Behavioural activation holds promise to reduce the global burden of depression as a treatment approach that is effective, easy to teach, scalable and acceptable to providers and patients across settings and cultures. This editorial reviews the history of behavioural activation, what it is, current evidence for its use and future directions.

\section{Declaration of interest}

J.W.K. provides professional training on behavioural activation.
Jonathan Kanter (pictured) is Associate Professor, Director of the Depression Treatment Specialty Clinic and Core Scientist with the Center for Applied Behavioural Health Research at the University of Wisconsin-Milwaukee. Ajeng Puspitasari, Maria Santos and Gabriela Nagy are clinical psychology graduate students in the Department of Psychology, University of WisconsinMilwaukee.

\section{Behavioural activation: history, evidence, and promise}

In 2008 the World Health Organization projected that by 2030 depression will be the most burdensome disease in the world. ${ }^{1}$ To address this immense and growing public health imperative, we need approaches to treating depression based on well-specified theories that facilitate scientific investigation across psychological and biological levels of analysis, and that are effective, easy to teach, scalable and acceptable to providers and patients across diverse settings and cultures. We suggest that behavioural activation holds great promise in this regard, representing an important component of a multifaceted clinical and scientific strategy to reduce the global burden of depression.

\section{History of behavioural activation}

Behavioural activation is a behavioural psychotherapy approach initially developed in the 1970s by Lewinsohn and colleagues. ${ }^{2,3}$ From its inception, the conceptual foundation of behavioural activation in simple behavioural learning principles, emphasised parsimony and efficiency of training. Early behavioural activation, which conceptualised depression as resulting from a broad deprivation of positive reinforcement and focused on identifying and scheduling pleasurable activities to increase contact with sources of positive reinforcement, accumulated considerable empirical support. Despite this support, behavioural activation fell out of favour by the 1980s, due in part to dissatisfaction with behaviour therapy's neglect of cognitive constructs that were seen as important to depression, and cognitive therapy for depression ascended in popularity. In fact, cognitive therapy included the primary behavioural techniques of activity monitoring and activity scheduling in its treatment package, but these techniques were employed in the framework of a cognitive change model rather than a reinforcement-based model.

Interest in behavioural activation as a viable stand-alone treatment was renewed by a component analysis of cognitive therapy that found that its behavioural techniques, performed in isolation from the larger cognitive therapy package, produced equivalent outcomes to the cognitive therapy package at the end of acute treatment and at a 2-year follow-up. ${ }^{4}$ Investigators concluded that behavioural activation techniques were potentially preferable on the grounds of improved efficiency and the ease of training therapists. Since then variants of behavioural activation have been researched more extensively, leading to the conclusion that it represents a well-established and efficacious treatment. ${ }^{5}$

Several variants of behavioural activation have been developed and refined over the years. ${ }^{3}$ Variants consistently focus on activity scheduling to obtain positive reinforcement but the manner in which this is achieved varies somewhat among approaches, and a variety of techniques to supplement and maximise the effectiveness of activity scheduling exist. Unlike earlier behavioural activation variants, behavioural activation treatments no longer focus solely on the experience of pleasant or pleasurable events (i.e. rewards) as a means of obtaining reinforcement. Instead, reinforcement is defined broadly as any environmental events that make healthy, antidepressant behaviour more likely. The therapist attempts to help the client re-engage in life in multiple ways, including increasing experiences of pleasure, activating 'approach behaviour' in the presence of strong cues for avoidance and aversive emotion, solving problems, engaging in behaviours that lead to experiences of mastery and accomplishment, and engaging in meaningful and difficult behaviours that are consistent with life values. Also unlike earlier variants, current approaches do not ignore cognition, but whereas cognitive therapy focuses on negative cognitive content, current behavioural activation focuses on the behaviour of ruminating and seeks to activate alternative healthy behaviours when a patient is ruminating.

\section{Evidence for behavioural activation}

Current approaches to behavioural activation have achieved considerable research attention. ${ }^{5}$ A notable, well-designed, large, randomised placebo-controlled trial compared a 24-session protocol of behavioural activation with cognitive therapy and paroxetine. This study found that all treatments performed well for milder depression, but for moderate-to-severe depression both behavioural activation and paroxetine outperformed cognitive therapy at the end of acute treatment at 24 weeks, ${ }^{6}$ and behavioural activiation was equivalent to continued paroxetine at 2-year follow-up. Overall, this study suggested that behavioural activation is an effective treatment for moderate-tosevere depression, producing equivalent efficacy to paroxetine with good treatment engagement, retention, efficiency and costeffectiveness. 
Concurrently, there is much interest in behavioural activation's applicability, the ease of training therapists, ease of implementation and acceptability by providers and patients across diverse treatment settings and target conditions. ${ }^{2}$ Populations in which behavioural activation research has been or is being conducted with promising results include individuals with depression comorbid with a variety of conditions (post-traumatic stress disorder, substance misuse, obesity, diabetes and cancer), patients of different ages (adolescents, college students, women with perinatal depression, elderly people in nursing homes and independent living contexts) and patients from different religious (for example Muslims in the UK) and ethnic backgrounds (for example individuals of Latin American origin in the USA). Behavioural activation has also shown promise across a variety of settings such as traditional mental health settings, exercise classes, hospitals and veteran's hospitals, in-patient medical and substance misuse units and community, public mental health settings.

In several of these research projects the service providers were not highly trained and included non-mental health specialists and para-professionals. The best example of this is the randomised trial by Ekers and colleagues, published in this Journal, ${ }^{7}$ in which mental health nurses with no previous psychotherapy training received training in behavioural activation and then delivered it effectively in a primary care setting, with statistically significant improvements in depression, overall functioning and satisfaction compared with usual care. The success of these investigators in training nurses (with no previous formal therapy training) is important and the cost-effectiveness analyses from this study suggested that behavioural activation may offer a lower cost per unit of improvement compared with other interventions aimed at improving treatment for depression in primary care.

\section{The promise of behavioural activation}

Behavioural activation's theory of pathology and change is well specified, with measures developed to evaluate each element; ${ }^{8}$ thus, behavioural activation is not only pragmatic and effective at the clinical level but it is testable at the level of bio-behavioural translational science. The assumption behind behavioural activation's direct behaviour change approach is not that behavioural factors are the only factors that are relevant to depression but that depression is a multisystem disorder and behavioural change, in the context of a genuine, empathic and validating therapeutic relationship, is a direct and pragmatic method for affecting the system. Thus, research has shown that both important cognitive changes ${ }^{4}$ and neurobiological changes ${ }^{9}$ occur after a successful course of treatment. Behavioural activation's theory in fact dovetails well with neurobiological research on the brain's reward circuitry and depression, in that environmental reinforcement and the circuitry of reward may be seen as parallel processes at different levels of analysis. This allows for translational research to identify biological, demographic, behavioural and environmental mediators and moderators of treatment outcome. More research is needed in these areas to elucidate its mechanism at behavioural and biological levels, explore its mechanism in the context of non-specific mechanisms (such as the therapeutic relationship) that also may contribute to a positive outcome, and tailor treatment strategies to maximise the implementation of the mechanism and its public health impact.

Another potential strength of this treatment is that its rationale is quite flexible, adaptable and acceptable and may be portable to traditionally hard-to-reach populations and regions. As presented to patients, behavioural activation's rationale that depression is not a function of inner causes may be less stigmatising than biological narratives about depression. ${ }^{10}$ This may be a particular strength of behavioural activation in terms of reaching underrepresented populations, for whom stigma may be a particular obstacle to treatment, as well as those for whom the medical model of depression may be foreign or threatening to local cultural beliefs. Behavioural activation, although rooted firmly in a Western medical tradition, does not need to be explained in Western medical terms to patients. Furthermore, competent behavioural activation therapists should be receptive to the patient's particular cultural values when defining activation targets, including culturally, spiritually and personally meaningful targets. In this way, it provides a depression treatment strategy that may improve outcomes and reduce the global disease burden while accommodating rather than challenging or requiring change in local cultural beliefs and practices. Additional research in these areas will be of substantial benefit.

Of great need is research that capitalises on behavioural activation's potential strengths in terms of parsimony, portability and efficiency of training and that explores how to effectively train clinicians in behavioural activation with attention to the types and amounts of resources needed for training. At the moment, some research exists to support the notion that effective behavioural activation is relatively easy to learn, but this research is still in its infancy and is far from definitive. In addition, some variants of behavioural activation may be easier to learn than others, and in fact the variant of it that has received the most empirical support ${ }^{6}$ may possibly be the most complex and difficult to learn. Thus, research on strategies incorporating modern technology (i.e. computers and the internet) for effective wide-scale training and dissemination of behavioural activation is necessary. Perhaps the ultimate promise of behavioural activation is that training protocols can be developed that maximise successful uptake and implementation of it with fidelity to its mechanism across diverse providers, settings and patients while minimising use of training resources. If such efficient and effective dissemination can be achieved, behavioural activation may prove to have a major impact on the global public health burden of depression.

Jonathan W. Kanter, PhD, Ajeng J. Puspitasari, BA, Maria M. Santos BA,
and Gabriela A. Nagy, BA, Department of Psychology, University of WisconsinMilwaukee, USA

Correspondence: Jonathan W. Kanter, PO Box 413, University of WisconsinMilwaukee, Milwaukee, WI 53201, USA. Email: jkanter@uwm.edu

First received 19 Oct 2011, final revision 8 Jan 2011, accepted 17 Jan 2012

\section{References}

1 World Health Organization. The Global Burden of Disease: 2004 Update. WHO, 2008.

2 Dimidjian S, Barrera Jr M, Martell C, Muñoz RF, Lewinsohn PM. The origins and current status of behavioural activation treatments for depression. Annu Rev Clin Psychol 2011; 7: 1-38.

3 Kanter JW, Manos RC, Bowe WM, Baruch DE, Busch AM, Rusch LC. What is behavioural activation? A review of the empirical literature. Clin Psychol Rev 2010; 30: 608-20.

4 Jacobson NS, Dobson KS, Truax PA, Addis ME, Koerner K, Gollan JK, et al. A component analysis of cognitive behavioural treatment for depression. J Consult Clin Psychol 1996; 64: 295-304.

5 Mazzucchelli T, Kane R, Rees C. Behavioural activation treatments for depression in adults: a meta-analysis and review. Clin Psychol Sci Pract 2009; 16: 383-411.

6 Dimidjian S, Hollon SD, Dobson KS, Schmaling KB, Kohlenberg RJ, Addis ME, et al. Randomized trial of behavioural activation, cognitive therapy, and antidepressant medication in the acute treatment of adults with major depression. J Consult Clin Psychol 2006; 74: 658-70. 
7 Ekers D, Godfrey C, Gilbody S, Parrott S, Richards DA, Hammond D, et al. Cost utility of behavioural activation delivered by the non-specialist. Br J Psychiatry 2011; 199: 510-1.

8 Manos RC, Kanter JW, Busch AM. A critical review of assessment strategies to measure the behavioural activation model of depression. Clin Psychol Rev 2010; 30: 547-61.
9 Dichter GS, Felder JN, Petty C, Bizzell J, Ernst M, Smoski MJ. The effects of psychotherapy on neural responses to rewards in major depression. Biol Psychiatry 2009; 66: 886-97.

10 Rusch LC, Kanter JW, Brondino MJ, Weeks CE, Bowe WM. Biomedical stigma reduction programs produce negative but transient effects on a depressed low-income community sample. J Soc Clin Psychol 2010; 29: 1021-31.

\section{poems \\ by doctors}

\title{
Why Did You Become a Doctor?
}

\section{Miles Burrows}

\author{
I originally wanted to be a witch \\ Sitting inside a Leiden jar \\ In the pathology museum \\ Or manifesting suddenly in a wardrobe \\ Or a trick of the light in a motorway café.
}

My fallback application

Was to become one of those nuns in La Dolce Vita

In an enormous starched wimple

Like the collar of Phillip II

As if my head was something that had fallen into a serviette.

I wanted to be Dr Zhivago

And have a Russian mistress on a sledge.

There are no crosswords in heaven

Because there is no tomorrow for the answer.

Later, psychiatry took my fancy.

I wanted to rescue an enchanted mad princess

From a tower in a wood

Where the senior registrar was making curry.

I hoped to meet some brilliant eccentric

Cataloguing shadows, or decanting clouds like Harpic

Into old sherry bottles.

The works of Freud were like a prolonged

Businessman's lunch in a German restaurant

Where the waiters have aprons that reach down to their ankles

And there is only one course on the menu but it is very good.

| was looking for an Irish country hour, Grade || listed,

Set in parkland, where the Medical Director

Organised an annual rough shooting party

For the staff to take potshots at each other

From behind hayricks

While inside the building huge women sat in stone circles

And we taunted them like boys taunting a dolmen.

Or the Persian king whipping the sea.

Sitting in the water tower on night duty

Waiting for the arrival of the princess

I found further philosophers plied their antique charms.

Minswanger, Glogg, Jaspers,

Kleist, Heidegger, Snoek

opened their swing doors to me

Like bistros in Charlotte Street with irresistible names.

And I would conjure with their smoky names

Savouring their names like baroque Italian ice creams

Or like a harpsichordist

Who slowly releases his fingers from the keys

And looks up at the candlelit ceiling

As if the music were someone he had left behind.

This poem is from The Hippocates Prize 2010: The Winning and Commended Poems, published by The Edge Press.

Chosen by Femi Oyebode. 